\title{
MaVIS: Media-aware Video Streaming Mechanism
}

\author{
Sunhun Lee and Kwangsue Chung \\ School of Electronics Engineering, Kwangwoon University, Korea \\ sunlee@adams.kw.ac.kr, kchung@kw.ac.kr
}

\begin{abstract}
Existing streaming protocols have no consideration for the characteristics of streaming applications because they only consider the network stability. In this paper, in order to overcome limitations of the previous schemes for video streaming, we propose a new video streaming mechanism called "MaVIS (Media-aware Video Streaming)". The MaVIS takes more sophisticated mechanism that considers both network and user requirements. Proposed mechanism improves the network stability by adjusting the sending rate suitable for current network state and it also provides the smoothed playback by preventing buffer underflow or overflow. Moreover, it is designed to consider characteristics of the video stream. Through the simulation, we prove that the MaVIS mechanism efficiently uses the buffer resources and provides improved network stability and smoothed playback.
\end{abstract}

\section{Introduction}

The Internet has recently been experiencing an explosive growth in the use of audio and video streaming applications. Such applications are delay-sensitive, semi-reliable and rate-based. Thus they require isochronous processing and QoS (Quality-of-Service) from the end-to-end viewpoint. However, today's Internet does not attempt to guarantee an upper bound on end-to-end delay and a lower bound on available bandwidth. As a result, most of video streaming applications use UDP (User Datagram Protocol) as a transport protocol that has no congestion control mechanism. However, the emergence of non-congestion-controlled streaming applications threatens unfairness to competing TCP (Transmission Control Protocol) traffic and possible congestion collapse [1].

Studies on the congestion controlled streaming protocol has been increasingly done since the 1990s $[2,3,4,5,6,17,8,9,10,11,12,13$. . These works attempt to guarantee the network stability and fairness with competing TCP traffic. However, by considering only the network stability, these works ignore the quality of video stream which is served to the end user. Moreover, most of existing streaming protocols have no consideration for the characteristics of video stream which affects significantly on the quality of streaming services.

In this paper, we propose a new video streaming mechanism called "MaVIS (Media-aware Video Streaming)". The MaVIS adjusts sending rate of video stream based on the current network state and controls the quality of video 
stream based on the receiver's buffer occupancy. It also considers the media characteristics of video stream which is requested from the end user. Therefore, the MaVIS maintains the network stability accomplished by previous works and achieves the smoothed playback by preventing the buffer underflow or overflow.

The rest of this paper is organized as follows. In Section 2, we review and discuss some of the previous works and in Section 3, we present the concept and algorithms introduced in the MaVIS mechanism. Detailed description of our simulation results are presented in Section 4. Finally, Section 5 concludes the paper and discusses some of our future works.

\section{Related Works}

While data applications such as Web and FTP (File Transfer Protocol) are mostly based on TCP, multimedia streaming applications will be based on UDP due to its real-time characteristics. However, UDP does not support congestion control mechanism. For this reason, UDP-based streaming protocols cause the unfairness with competing TCP traffic and the starvation of congestion controlled TCP traffic which reduces its bandwidth share during overload situation. To overcome this limitation, several congestion controlled streaming protocols have been developed, recently. These works mostly interested in network stability. For the network stability, they apply the congestion control mechanism to the non-congestion controlled streaming protocol, such as UDP, or modify the existing congestion control mechanism in consideration of real-time characteristics of video streaming.

Previous works, interested in the network stability, can be further categorized into three different approaches. The first approach mimics TCP's AIMD (Additive Increase Multiplicative Decrease) algorithm to achieve the TCP-friendliness $[2,3,4,5,6$. The second approach adjusts its sending rate according to the Padhye's TCP throughput modeling equation [7, 8, 9, 10]. The third approach uses the bandwidth estimation scheme that was employed in the TCP Westwood [11, 12 .

A typical example is the TFRC (TCP-Friendly Rate Control) for unicast streaming applications which uses the TCP throughput modeling equation [14]. Padhye et al. presents an analytical model for the available bandwidth share $(T)$ of TCP connection with $S$ as the segment size, $p$ as the packet loss rate, $t_{R T T}$ as the RTT (Round Trip Time), $t_{R T O}$ as the RTO (Retransmission Time-Out). The average bandwidth share of TCP connection depends mainly on $t_{R T O}$ and $p$ as shown in (1):

$$
T=\frac{S}{t_{R T T} \sqrt{\frac{2 p}{3}}+t_{R T O}\left(3 \sqrt{\frac{3 p}{8}}\right) p\left(1+32 p^{2}\right)}
$$

This approximation is reasonable for reaching TCP-friendliness. For this reason, many TCP-friendly rate control schemes use this analytical model.

The TFRC with Padhye's modeling equation is responsive to network congestion over longer time period and changes the sending rate in a slowly responsive 
manner. Therefore, it can work well with TCP flows and reduce the packet loss occurrence. But it has some limitations which are oscillations of sending rate and possibility of buffer underflow or overflow. It also has no consideration for the quality of streaming service. The improved network stability does not guarantee an improved quality of service on video streaming.

Unlike the network viewpoint protocol, discussed above, the buffer-driven scheme is a streaming protocol which provides smoothed playback of video stream to the user. Usually, streaming applications use a buffer to compensate delay and jitter caused from the network. The buffer-driven scheme controls the video quality and schedules data transmission based on receiver and sender buffer occupancies. Therefore, it provides smoothed playback to the user by preventing buffer underflow or overflow. However, the buffer-driven scheme still has instability and unfairness problems because it has no concern about the network state 13 .

Figure 1 shows the existing streaming protocols which have been classified into four different approaches. Existing streaming protocols control the transmission rate of the video stream by using (1) the modified congestion control mechanism, (2) the Padhye's TCP throughput modeling equation, (3) the estimation of available network bandwidth, and (4) the buffer occupancy. The MaVIS mechanism, proposed in this paper, takes two approaches together to achieve both the network stability and the smoothed playback, simultaneously.

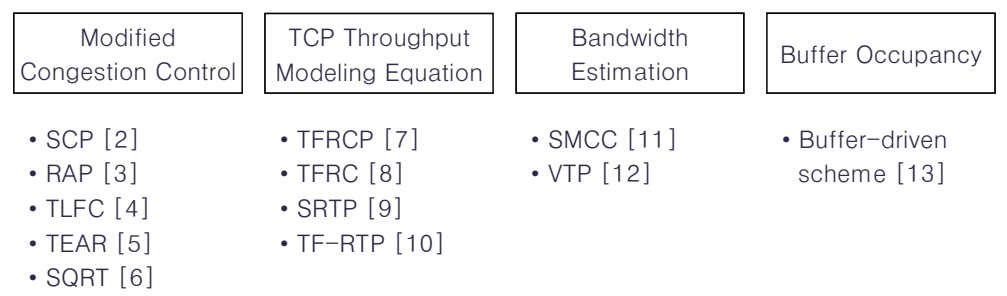

Fig. 1. Classification of existing streaming protocols

\section{MaVIS Mechanism}

\subsection{Overall Architecture}

The existing streaming protocols mostly interested in network stability. To improve the network stability, they control the sending rate of video stream according to the current network state. However, for the efficient video streaming service over Internet, streaming protocol has to provide the smoothed playback to the user by preventing buffer underflow or overflow. Moreover, to improve the quality of streaming service, it has to consider the media characteristics of video stream. 
We propose a new video streaming mechanism that controls the sending rate of video stream based on current network state and adjusts the video quality based on current receiver buffer occupancy. It also efficiently manages the buffer resource on the basis of the characteristics of video stream, typically an encoding rate of video stream. Figure 2 shows the overall architecture of the proposed mechanism. Basically, the MaVIS mechanism relies on the RTP/RTCP (Realtime Transport Protocol/Realtime Transport Control Protocol) model which is standardized by IETF (Internet Engineering Task Force). The RTCP periodically reports the information on network state to the sender [15]. According to this feedback information, the MaVIS determines the network state as congestion_state or stable_state.

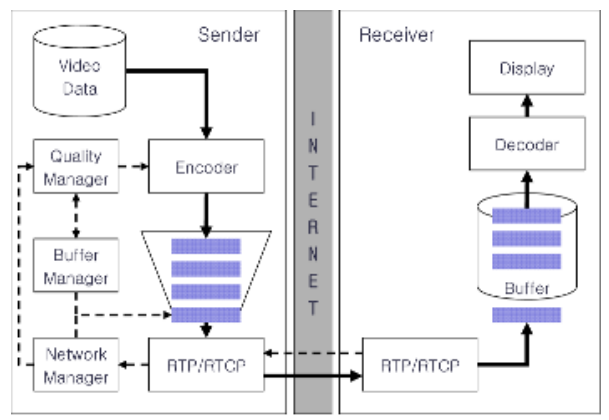

Fig. 2. Overall architecture

After deciding the network state, the MaVIS estimates the receiver buffer occupancy at a sender side. Then it controls the sending rate or the video quality on the basis of the current network state and buffer occupancy. If the estimated receiver buffer occupancy is very high or low, then it controls the video quality to prevent buffer underflow or overflow. Otherwise, it controls the sending rate for network stability according to the current network state.

\subsection{Decision on Network State}

The MaVIS decides the network state based on the packet loss rate which can be calculated from the RR (Receiver Report) of RTCP. The packet loss rate is estimated in a receiver side by checking the sequence numbers included in RTP header of the data packets. It is calculated on the proportion of the number of packets received to the number of packets sent during the RTCP interval [15].

$$
\text { Packet loss rate, } p=1-\frac{\text { Number of packets received }}{\text { Number of packets sent }}
$$

Figure 3 shows the decision rule for network state. If the packet loss rate is zero, the network state is decided to be stable_state. Otherwise, the network 
state is decided to be congestion_state by assuming that the packet losses are caused by congestion.

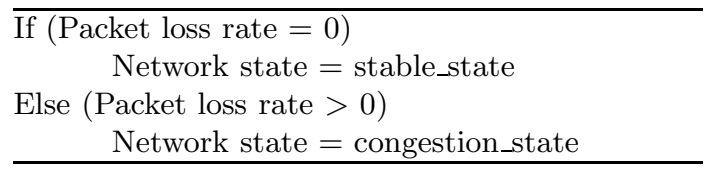

Fig. 3. Decision rule for network state

\subsection{Estimation on Receiver Buffer Occupancy}

To provide smoothed playback, the MaVIS is designed to prevent buffer underflow or overflow. For this purpose, the MaVIS controls the video quality by estimating the receiver's buffer occupancy.

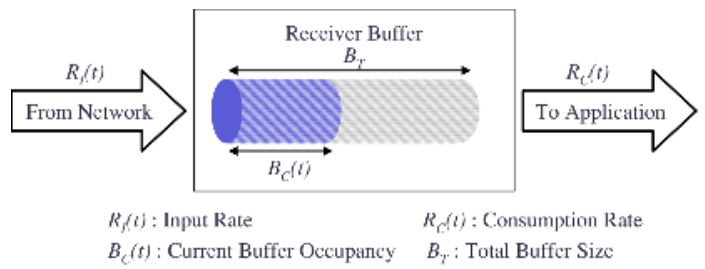

Fig. 4. Buffer state in a receiver side

Figure 4 shows the buffer state in a receiver side with $B_{T}$ as the total buffer size, $B_{C}(t)$ as the current buffer occupancy, $R_{I}(t)$ as the input data rate in a receiver. If we ignore the network delay, $R_{I}(t)$ is equal to the outgoing rate in a sender. $R_{C}(t)$ is the consumption rate by streaming application at a receiver side, equal to the encoding rate of video stream. The sender can estimate the receiver buffer occupancy $\left(B_{E}\right)$ as follows:

$$
\begin{gathered}
B_{E}(t+1)=B_{C}(t)+\left\{R_{I}(t)-R_{C}(t)\right\} \times R T T \\
B_{C}(t) \approx B_{E}(t) \\
R_{I}(t)=\frac{\text { Number of packets } \times \text { Packet size }}{\text { RTCP interval }} \\
R_{C}(t)=\text { Encoding rate at time }(\mathrm{t})
\end{gathered}
$$

If the receiver buffer occupancy is estimated to be underflow or overflow, encoding rate of video stream is changed. After changing the next video quality, $B_{C}(t)$ is re-calculated based on the changed encoding rate. In (3), the MaVIS 
Table 1. Control scheme in MaVIS

\begin{tabular}{|c|c|c|}
\hline \multirow{2}{*}{$\begin{array}{c}\text { Buffer } \\
\text { occupancy }\end{array}$} & congestion_state & stable_state \\
\cline { 2 - 3 } & Network state \\
\hline \hline MinTH MinTH\% $\sim$ MaxTH\% & Decrease video quality & Decrease video quality \\
\hline MaxTH $\sim 100 \%$ & Increase video quality & Increase video quality \\
\hline
\end{tabular}

predicts one-RTT-early the receiver buffer occupancy. This approach efficiently prevents buffer underflow or overflow with no additional overhead in high delay networks.

The management of buffer resource in MaVIS takes a characteristic of video stream into consideration. Encoding rate of video stream, determined by using a video compression scheme, is a representative characteristic of video stream, because it has an effect on change of network conditions and buffering level. To efficiently manage the buffer resource, MaVIS defines two important thresholds which prevent the buffer underflow or overflow. These thresholds divide the operation of MaVIS between the response to network state and the response to buffer occupancy. A threshold with no consideration of the encoding rate causes an unnecessary change of sending rate and video quality that can be annoying to the user. To overcome this problem, the MaVIS sets these thresholds based on the encoding rate of video stream. These appropriate thresholds will help to improve efficiency of buffer resources. It also reduces unnecessary change of sending rate and video quality. The MaVIS sets the thresholds as shown in (7). $R_{E N C}$ is the encoding rate of video stream and $B_{T}$ is total receiver buffer size. These two thresholds are set to the sufficient buffering level which does not cause either buffer underflow or overflow for one second.

$$
\operatorname{MinTH}(\%)=\frac{R_{E N C}(\text { bps }) / 8 \text { bits }}{B_{T}(\text { bytes })} \times 100, \operatorname{MaxTH}(\%)=100-\text { MinTH }
$$

\subsection{Sending Rate and Video Quality Control}

The MaVIS firstly determines the network state as congestion_state or stable_state based on the packet loss rate. And then it estimates the receiver buffer occupancy. Based on the network state and the buffer occupancy, it either controls the sending rate or the video quality.

Table 1 shows the control scheme that adjusts sending rate or video quality based on network state and buffer occupancy. For the congestion_state, the sender has to reduce the sending rate for the network stability. But, if the receiver buffer occupancy is estimated as $(0 \sim M i n T H \%)$, we decreased the video quality to prevent buffer underflow without adjusting the sending rate. For (MaxTH $\sim 100 \%$ ) of buffer occupancy, the video quality is increased to prevent the buffer overflow with no sending rate control. However, in case of (MinTH $\sim$ MaxTH\%), it simply reduces the sending rate by $\alpha$ for the network stability. 
For $(0 \sim \operatorname{MinTH\% })$ of the buffer occupancy in the stable_state, the sender only decreases the video quality. However, for $($ MaxTH $\sim 100 \%)$, it increases the video quality. In case of (MinTH MaxTH\%), the MaVIS scheme increases the sending rate by $\beta$ for competing with other traffics.

Parameter $\alpha$ and $\beta$ are derived from the congestion control mechanism need in conventional TCPs, to improve fairness with competing TCP traffics. From 8], it is found that the overall TCP bandwidth share is inversely proportional to the square root of the packet loss rate. Hence, after receiving a loss notification from the receiver, the sender can reduce its sending rate by $\alpha$ from the current sending rate, $R_{C}$.

$$
\alpha=R_{C} \times \sqrt{p}
$$

For the case of stable_state, the sending rate can be increased by parameter, $\beta$. This increase value does not exceed the increase of the competing TCP connection under the same network conditions. Thus, the sender has to adjust the sending rate to be equal to the competing TCP connection, in order to improve the fairness. For this requirement, the MaVIS scheme increases sending rate by $R_{\text {Inc }}$ at each RTCP interval instead of each RTT.

$$
\beta=\frac{R_{I n c}}{t_{R T T}}, \text { where } R_{I n c}=\frac{\text { RTCP interval }}{t_{R T T}}
$$

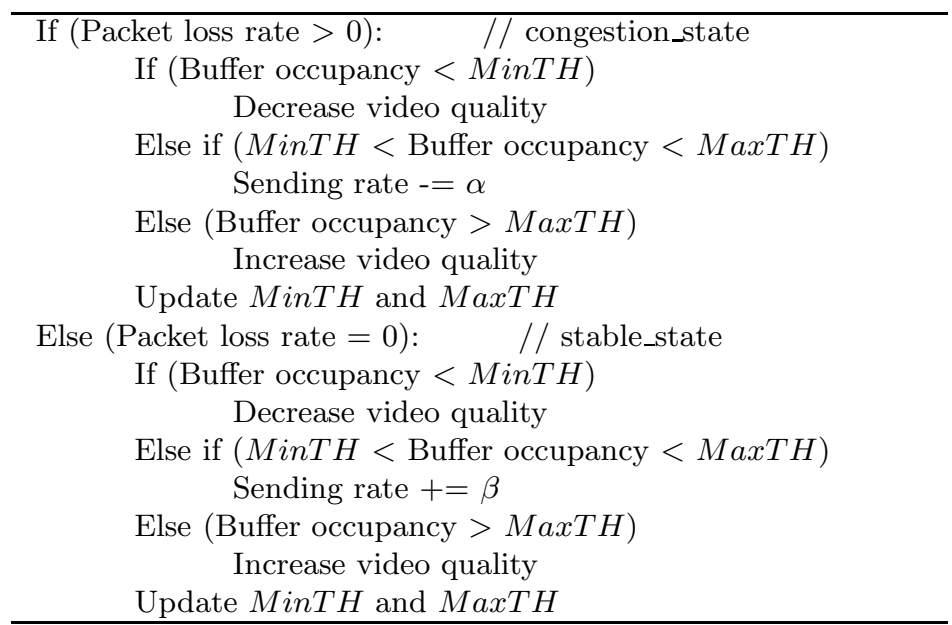

Fig. 5. MaVIS's algorithm

Figure 5 shows the pseudo code of our MaVIS mechanism. Depending on the network state and estimated buffer occupancy, we decide whether the sending rate or the video quality should be controlled. If the estimated receiver buffer occupancy is very high or low, then the MaVIS controls the video quality to 
prevent buffer underflow or overflow. Otherwise, the MaVIS controls the sending rate for the network stability according to the current network state. Since the estimation of receiver buffer occupancy is related to the encoding rate of video stream, two threshold values must be updated accordingly, if the video quality is changed.

\section{Simulation and Evaluation}

\subsection{Simulation Environment}

In this Section, we present our simulation results. Using the ns- 2 simulator, the performance of the MaVIS mechanism has been measured, compared with the TFRC and the buffer-driven scheme [16]. To emulate the competing network conditions, background TCP Reno traffics were introduced.

Figure 6] shows the network topology for our simulations. We assume that the video quality is controllable by using the scalable video codec. Under this assumption, five different video qualities are used, such as $20 \mathrm{Mbps}, 16 \mathrm{Mbps}$, $12 \mathrm{Mbps}$, $8 \mathrm{Mbps}$, and $4 \mathrm{Mbps}$. $8 \mathrm{Mbytes}$ buffer space is allocated for the receiver buffer. We assume that the initial buffer occupancy is $50 \%$.

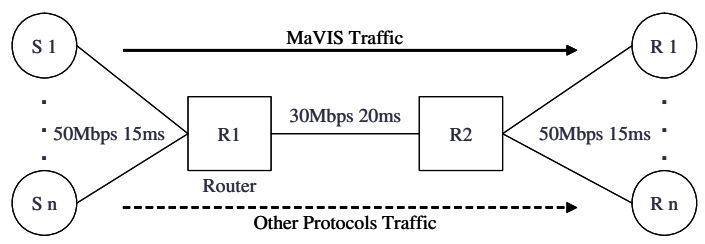

Fig. 6. Simulation environment

\subsection{Performance Evaluation}

Before discussing the performance of the MaVIS mechanism, we first examine the accuracy of the buffer estimation algorithm. Because the MaVIS controls either the sending rate or the video quality based on the estimated buffer occupancy, the accuracy of estimation algorithm significantly affects the performance of our mechanism. Figure 7 compares the estimated buffer occupancy in a sender side and the actual one in a receiver side. It shows that the estimated buffer occupancy and the actual one are very close. It is also shown in Fig. 7 that the media-aware thresholds are adaptively changed based on encoding rate of the video stream.

To evaluate our proposed mechanism, throughput, video quality, buffer occupancy, and packet losses are measured. Figure 8 (a) shows that the MaVIS dynamically controls the sending rate and the video quality. In the beginning, the sender transmits the video stream with 16Mbps. At about 21, 56, 80 and 102 second, the sender increases the video quality in order to prevent buffer overflow. 


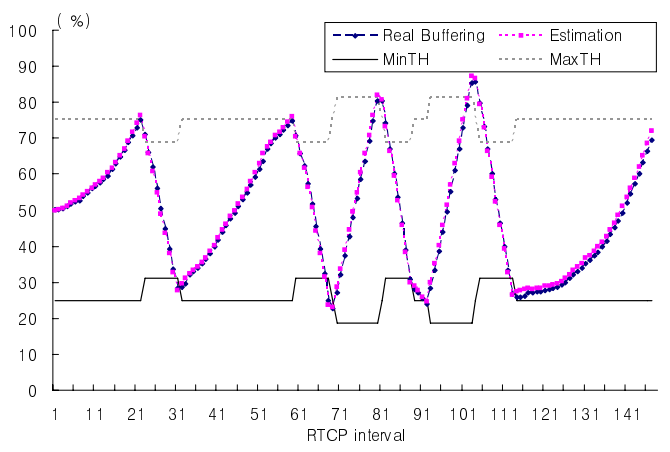

Fig. 7. Estimation of receiver buffer occupancy

At about 30, 68, 85, 91 and 112 second, the sender decreases the video quality in order to prevent buffer underflow. In these cases, the sender only controls the video quality without the sending rate control. Figure 8 (b) shows the packet losses of the MaVIS mechanism, compared with RTP. Unlike RTP, the MaVIS can reduce packet losses by controlling the sending rate based on current network state. Approximately, the MaVIS mechanism reduces $40 \%$ of packet losses, compared with RTP.

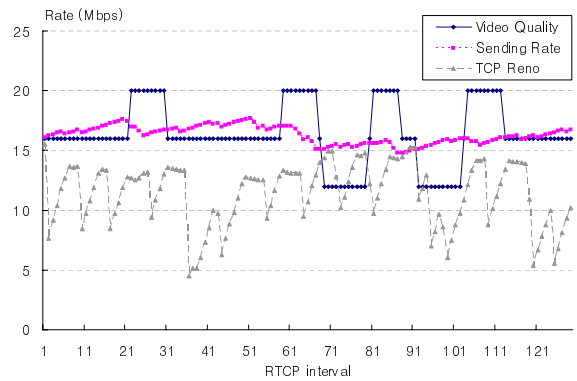

(a) Throughput and video quality

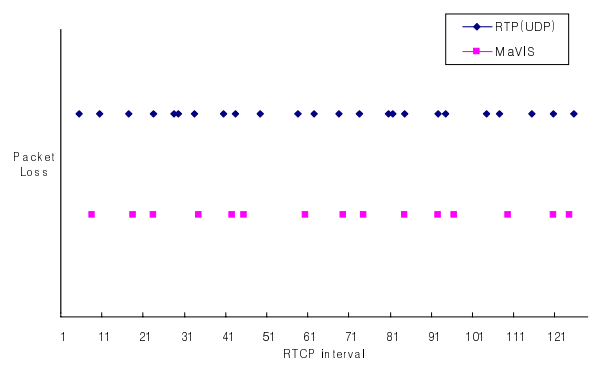

(b) Packet losses

Fig. 8. Performance of the MaVIS mechanism

In Fig. 9, the MaVIS is compared with the previous streaming protocols, the TFRC and the buffer-driven scheme in terms of the packet losses and the buffer occupancy. Figure 9 (a) shows the changes in the buffer occupancy. The TFRC experiences a serious buffer underflow, because it has no control on the video quality upon the buffer occupancy. However, the MaVIS mechanism and the buffer-driven scheme are successfully preventing the buffer underflow or overflow by adaptively controlling the video quality. In Fig.9(b), the packet losses for each 
protocol are shown. Because the buffer-driven scheme controls the video quality based on the current buffer state without the consideration of the network state, it suffers more packet losses than our proposed mechanism. However, the packet losses in the MaVIS and the TFRC are about the same. From this result, it is shown that the MaVIS mechanism has the approximately same performance with the TFRC in the aspects of the network stability.

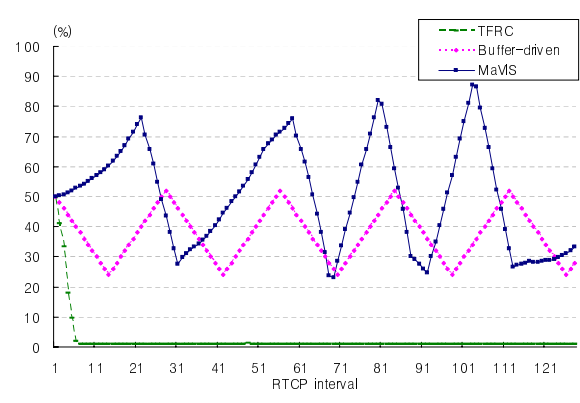

(a) Buffer occupancy

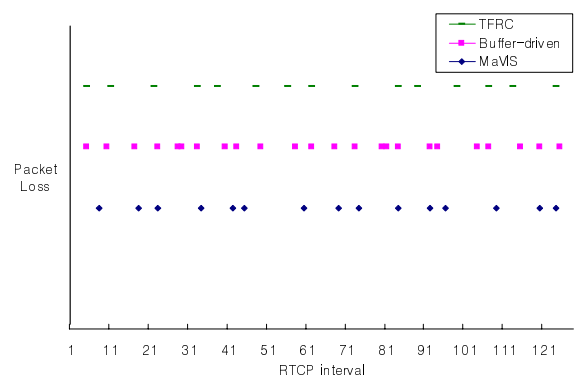

(b) Packet losses

Fig. 9. Comparison between MaVIS and previous works

\section{Conclusion}

In this paper, in order to overcome limitations of the previous streaming protocols, we propose a new mechanism for efficient video streaming, called MaVIS. The MaVIS mechanism considers both user and network requirements. It controls the sending rate or the video quality on the basis of both the estimated buffer occupancy and the network state. The MaVIS improves the network stability by reducing the packet losses and also provides the smoothed playback by preventing buffer underflow or overflow. Moreover, the MaVIS efficiently manages the buffer resource and reduces unnecessary sending rate and video quality changes because it is designed to consider the media characteristics of the video stream.

Simulation results have shown that our MaVIS mechanism has a better performance than previous approaches. In the future, we plan to apply the quality adaptation technique to our rate control scheme, as well as to perform real experiments in a real network environment.

\section{Acknowledgement}

This research was supported by the MIC(Ministry of Information and Communication), Korea, under the ITRC(Information Technology Research Center) support program supervised by the IITA(Institute of Information Technology Assessment). 


\section{References}

1. S. Floyd and F. Kevin: Router mechanisms to support end-to-end congestion control. Technical Report, LBL-Berkeley. (1997)

2. S. Cen, C. Pu, and J. Walpole: Flow and congestion control for internet streaming applications. Multimedia Computing and Networking. (1998)

3. R. Rejaie, M. Handley, and D. Estrin: RAP: An end-to-end rate based congestion control mechanism for real-time streams in the Internet. IEEE INFOCOMM. (1999)

4. S. Na and J. Ahn: TCP-like flow control algorithm for real-time applications. IEEE ICON. (2000)

5. I. Rhee, V. Ozdemir, and Y. Yi: TEAR: TCP emulation at receivers - flow control for multimedia streaming. Technical Report, NCSU. (2000)

6. D. Bansal, and H. Balakrishnan: Binomial Congestion Control Algorithms. IEEE INFOCOMM. (2001)

7. J. Padhye, J. Kurose, D. Towsley, and R. Koodli: A model based TCP-friendly rate control protocol. NOSSDAV. (1999)

8. S. Floyd, M. Handley, J. Padhye, and J. Widmer: Equation-based congestion control for unicast applications. ACM SIGCOMM. (2000)

9. B. Song, K. Chung, and S. Rhee: Distributed Transport Platform for TCP-friendly streaming. LNCS 2662, Springer-Verlag Press. (2003)

10. S. Lee and K. Chung: TCP-friendly rate control scheme based on RTP. International Conference on Information Networking. (2006)

11. N. Aboobaker, D. Chanady, M. Gerla, and M. Sanadidi: Streaming media congestion control using bandwidth estimation. IFIP/IEEE Internation Conference on Management of Multimedia Networks and Services. (2002)

12. A. Balk, D. Maggiorini, M. Gerla, and M. Sanadidi: Adaptive MPEG-4 video streaming with bandwidth estimation. QoS-IP. (2003)

13. D. Ye, X. Wang, Z. Zhang, and Q. Wu: A buffer-driven approach to adaptively stream stored video over Internet. International Conference on High Speed Networks and Multimedia Communications. (2002)

14. J. Padhye, V. Firoiu, D. Towsley, and J. Kurpose: Modeling TCP throughput: A simple model and its empirical validation. ACM SIGCOMM. (1998)

15. H. Schulzrinne, S. Casner, R. Frederick, and V. Jacobson: RTP: A transport protocol for real-time applications. IETF, RFC 1889. (1996)

16. UCB LBNL VINT: Network Simulator ns (Version 2). http://www-mash.cs.berkeley.edu/ns/ 